\title{
Laser-induced ultrafast spin dynamics in $\mathrm{ErFeO}_{3}$
}

\author{
J. A. de Jong, ${ }^{1}$ A. V. Kimel,,${ }^{1}$ R. V. Pisarev, ${ }^{2}$ A. Kirilyuk, ${ }^{1}$ and Th. Rasing ${ }^{1}$ \\ ${ }^{1}$ Radboud University Nijmegen, Institute for Molecules and Materials, NL-6525 AJ Nijmegen, The Netherlands \\ ${ }^{2}$ Ioffe Physical-Technical Institute, Russian Academy of Sciences, 194021 St. Petersburg, Russia \\ (Received 5 July 2011; revised manuscript received 9 August 2011; published 12 September 2011)
}

\begin{abstract}
Using 100-fs optical laser pulses, we have been able to excite and probe spin dynamics in the rare-earth orthoferrite $\mathrm{ErFeO}_{3}$. The investigation was performed in a broad temperature range with the focus on the vicinities of the compensation point $T_{\text {comp }} \approx 47 \mathrm{~K}$ and the spin reorientation transition region in the interval $86 \mathrm{~K} \lesssim T \lesssim 99 \mathrm{~K}$. Spin precession excited by the laser pulse was present in a large part of the investigated temperature range, but was especially strong near the spin reorientation region. In this region the laser pulse also caused an ultrafast spin reorientation. By changing the laser pulse fluence, we could vary both the reorientation amplitude and the reorientation speed. We show that the laser-induced spin dynamics in $\mathrm{ErFeO}_{3}$ is caused in part by heating and in part by the inverse Faraday effect. Comparing to the results of similar experiments in other rare-earth orthoferrites, we found the speed of the laser-induced spin reorientation to be significantly lower. We attribute this finding to the weaker electron-phonon coupling of the $\mathrm{Er}^{3+} 4 f$ electrons with the lattice.
\end{abstract}

DOI: 10.1103/PhysRevB.84.104421

PACS number(s): 75.78.Jp, 78.47.-p, 75.50.Ee, 78.20.Ls

\section{INTRODUCTION}

In the past years it has been shown that with the help of femtosecond laser pulses the magnetic order in condensed matter systems can be changed at a (sub)picosecond time scale. ${ }^{1}$ Laser-induced demagnetization, ${ }^{2}$ excitation of precession, ${ }^{3}$ orientation phase transitions, ${ }^{4}$ changes in magnetic anisotropy, ${ }^{5}$ and even complete reversal of magnetization ${ }^{6,7}$ have been observed. This has triggered a plethora of experimental and theoretical work and opened a new research area of magnetism. In contrast to metals, the rare-earth (RE) orthoferrites form a class of insulating materials that is very suitable for fast, laser-induced switching: being canted antiferromagnets, their spin dynamics is governed by the exchange interaction and is therefore about two orders of magnitude faster than in metallic or insulating ferromagnets. ${ }^{4}$

In the RE orthoferrites two mechanisms have been demonstrated by which a laser pulse affects the magnetization. The first is ultrafast heating in the vicinity of a spin reorientation transition (SRT). The SRT is a phase transition region which comprises two second-order phase transitions within a certain temperature interval, where the spins rotate by $90^{\circ}$ from one symmetry axis to another (see Fig. 1). In $\mathrm{TmFeO}_{3}$ ultrafast heating resulted in a rotation of the magnetization within a few picoseconds and the simultaneous excitation of one of the spin resonance modes. ${ }^{4,8}$ The second mechanism is based on the inverse Faraday effect (IFE): a circularly polarized laser pulse can generate a strong effective magnetic field in the material. ${ }^{9}$ This was shown to excite resonance modes in $\mathrm{DyFeO}_{3}, \mathrm{TmFeO}_{3}$, and $\mathrm{HoFeO}_{3}$ and to cause an inertia-driven spin rotation in $\mathrm{HoFeO}_{3}$ (Refs. 3,8, and 10).

The process of ultrafast heating and the subsequent spin reorientation is not completely understood. This is mainly because such a subpicosecond excitation brings the material into a strongly nonequilibrium state. From experiments with $\mathrm{TmFeO}_{3}$ we know only that the spin reorientation occurs on a time scale in the order of a quarter of the period of the antiferromagnetic precession, which is a few picoseconds. In the RE orthoferrites, the SRT is driven by a repopulation of electronic sublevels within the RE ground multiplet. ${ }^{11,12}$
However, the details of the electronic structure differ much between the various RE ions and the question is how this influences the ultrafast heating and the laser-induced spin dynamics.

$\mathrm{ErFeO}_{3}$ is very different from $\mathrm{TmFeO}_{3}$ in some important details in the electronic structure of the RE ion. First of all, $\mathrm{Er}^{3+}$ has an odd number of $4 f$ electrons (Kramers ion) and therefore the electronic states in the ground multiplet form doublets. ${ }^{13}$ In contrast, $\mathrm{Tm}^{3+}$ has an even number of $4 f$ electrons and its electronic states are isolated singlets. ${ }^{14}$ Also, the electron-phonon coupling between the RE $4 f$ electrons and the lattice is weaker in $\mathrm{ErFeO}_{3}$ than in $\mathrm{TmFeO}_{3}$ (Ref. 15). There are two temperature regions where ultrafast heating can induce magnetization dynamics in $\mathrm{ErFeO}_{3}$. The first is the SRT, which is of the same type as in $\mathrm{TmFeO}_{3}$ and in the same temperature range. The second is the magnetization

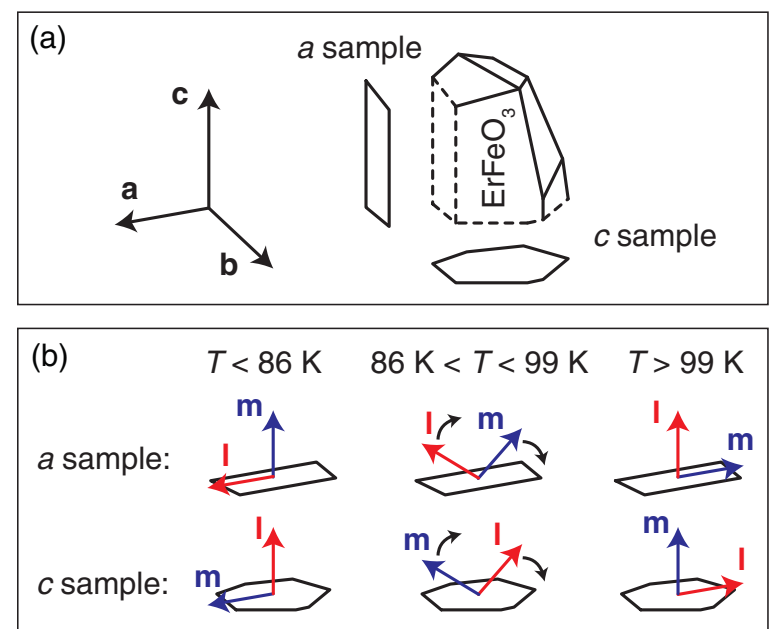

FIG. 1. (Color online) (a) The $a$ sample and $c$ sample were cut perpendicular to the $a$ axis and $c$ axis, respectively. (b) Orientation of the ferromagnetic vector $\mathbf{m}$ and antiferromagnetic vector $\mathbf{l}$ of the $\mathrm{Fe}^{3+}$ sublattices in the $a$ sample and $c$ sample at temperatures $T$ around the region of the spin reorientation transition. 
(a)

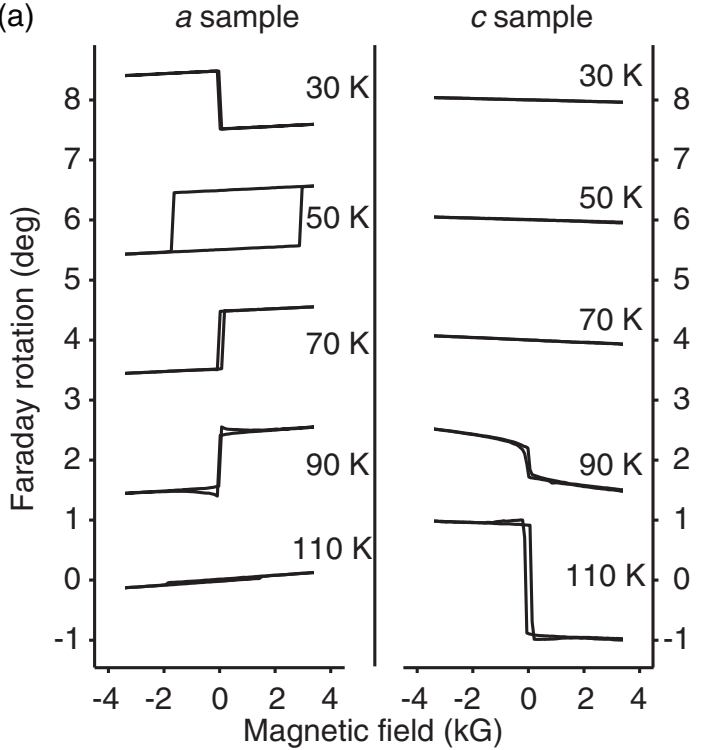

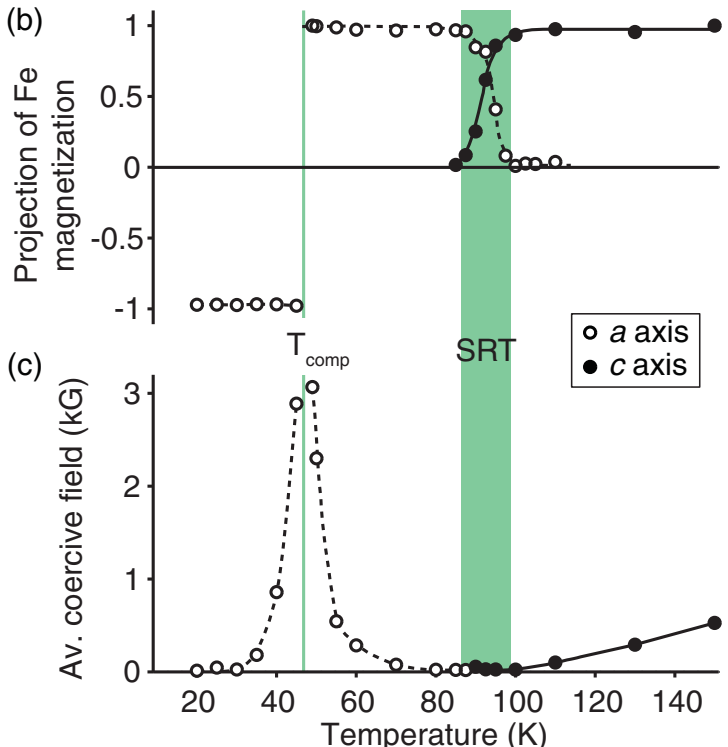

FIG. 2. (Color online) (a) Hysteresis of the magnetization in the $a$ sample (left) and $c$ sample (right) in response to a magnetic field applied perpendicular to the sample surface. With the help of the Faraday effect we probe the out-of-plane component of the magnetization. (b) Normalized magnetization of $\mathrm{Fe}^{3+}$ and (c) average coercive field along the $a$ axis (open dots) and the $c$ axis (filled dots). We identify the compensation point through the switch in the sign of the Fe magnetization and the diverging coercive field. In the spin reorientation transition the magnetization component along the $a$ axis vanishes, while it appears along the $c$ axis.

compensation point $T_{\text {comp }}$, which is the temperature where the magnetizations of the $\mathrm{Er}$ and Fe sublattices exactly cancel each other. The presence of a $T_{\text {comp }}$ in $\mathrm{ErFeO}_{3}$ is unique for the RE orthoferrites. In metallic ferrimagnets a magnetic compensation point is more common and it has been demonstrated that in its vicinity the magnetization can be reversed on a subpicosecond time scale. ${ }^{16,17}$

The goal of this article is to investigate the ultrafast laser-induced spin dynamics in $\mathrm{ErFeO}_{3}$ in the temperature interval that includes both the compensation point and the SRT. We show that at a temperature just below the SRT laser-induced heating leads to an ultrafast spin reorientation. Contrary to what was observed in similar experiments on $\mathrm{TmFeO}_{3}$, the reorientation takes longer than a few picoseconds. This is remarkable since heating of the lattice should take place in less than 500 fs. We will argue that not this heating, but the weak electron-phonon coupling between the lattice and the RE $4 f$ electrons limits the speed of the reorientation in $\mathrm{ErFeO}_{3}$. A consequence of this is that the reorientation speed depends on and can thus be controlled by the laser pulse fluence. Additionally, we report on the excitation of a spin resonance mode by an ultrashort laser pulse through both a heat-induced anisotropy change and the IFE.

\section{MATERIAL}

$\mathrm{ErFeO}_{3}$ forms crystals with an orthorhombically distorted perovskite structure (space group $D_{2 h}^{16}$ or Pbnm). The unit cell has three different axes: $a=5.3 \AA, b=5.6 \AA$, and $c=7.6 \AA$ (Ref. 18). As a consequence, $\mathrm{ErFeO}_{3}$ crystals are known to be moderately birefringent. ${ }^{19}$ The $\mathrm{ErFeO}_{3}$ crystal we used in our experiment was grown using the floating zone method. ${ }^{20}$ The crystallographic axes of the crystal were determined by x-ray diffraction. Two samples, both approximately $100-\mu \mathrm{m}$ thick, were cut perpendicular to the $a$ axis (further in the article we will refer to it as $a$ sample) and perpendicular to the $c$ axis (c sample), respectively [see Fig. 1(a)].

Similar to other RE orthoferrites, the optical properties of $\mathrm{ErFeO}_{3}$ in the visible and near infrared spectral range are dominated by electronic transitions from the ${ }^{6} A_{1 g}$ levels to the ${ }^{4} T_{1 g}$ and ${ }^{4} T_{2 g}$ levels in the $\mathrm{Fe}^{3+}$ ions. ${ }^{21-23}$ These transitions are visible as broad bands in the absorption spectrum. Being forbidden by the parity selection rule for electric-dipole transitions, they are characterized by moderate absorption coefficients in the range of several hundred $\mathrm{cm}^{-1}$ (Ref. 23). Nevertheless, such absorption is sufficient for inducing heat-driven ultrafast magnetic effects and orientation phase transitions as we show below. Moreover, on top of these there are groups of very narrow lines corresponding to $\mathrm{Er}^{3+}$ transitions from the ${ }^{4} I_{\frac{15}{2}}$ ground state term to higher terms. ${ }^{13,22}$ In some other RE orthoferrites these lines are broader, ${ }^{11}$ which is another sign that the electron-phonon coupling between the $\mathrm{Er}^{3+} 4 f$ electrons and the lattice is relatively weak. ${ }^{24}$ The ground state term is split into eight Kramers doublets by the crystal field. Because the $\mathrm{Er}^{3+}$ ion has an odd number of electrons, this degeneracy can only be lifted by magnetic interactions.

The magnetic properties of $\mathrm{ErFeO}_{3}$ are rather unusual. At $T_{\mathrm{N}}=643 \mathrm{~K}$ the $\mathrm{Fe}^{3+}$ ions order into four sublattices, which are antiferromagnetically coupled. Due to the DzyaloshinskiiMoriya interaction there is a small canting between these sublattices leading to a macroscopic magnetization. The result is a $\Gamma_{4}$ configuration, with a net magnetization along the $c$ axis. The $\mathrm{Er}^{3+}$ ions only order at temperatures lower than $4.4 \mathrm{~K}$ (Ref. 25). However, they are coupled antiferromagnetically to their $\mathrm{Fe}^{3+}$ neighbors by a superexchange interaction. This interaction is relatively weak and causes only a small splitting of the Kramers doublets. Therefore the $\mathrm{Er}^{3+}$ magnetization is 
negligible above $100 \mathrm{~K}$. As the temperature is lowered, however, it starts to increase. At the magnetization compensation point, around $45 \mathrm{~K}$, it is equal to the net magnetization of the $\mathrm{Fe}^{3+}$ sublattices and the total magnetization vanishes. ${ }^{25}$ The SRT results from the fact that the size of the splitting of the Kramers doublets increases if the magnetic configuration of the $\mathrm{Fe}^{3+}$ ions changes from $\Gamma_{4}$ to $\Gamma_{2}$, which has a net magnetization along the $a$ axis. ${ }^{22}$ At high temperatures the single ion anisotropy of the $\mathrm{Fe}^{3+}$ ions fixes the configuration to $\Gamma_{4}$. However, at low temperatures it becomes energetically more favorable to have a larger splitting of the Kramers doublets. The SRT region, around $90 \mathrm{~K}$, marks the boundary between these two [see Fig. 1(b)].

The compensation point and the SRT region in our samples were identified by measuring the hysteresis of the magnetization component perpendicular to the sample surface using the Faraday effect on a laser beam with the wavelength $\lambda=$ $633 \mathrm{~nm}$. Figure 2(a) shows some of the measured hysteresis loops. We plotted the remanences and the average coercive fields (half the width) measured from the loops in Figs. 2(b) and 2 (c), respectively. Due to the vanishing net magnetization, the coercive field diverges at the temperature $T_{\text {comp }}$. In our $a$ sample we find $T_{\text {comp }} \approx 47 \mathrm{~K}$. Because in $\mathrm{ErFeO}_{3}$ magnetooptical properties such as the Faraday effect are dominated by the $\mathrm{Fe}^{3+}$ ions, we are only sensitive to the magnetization of $\mathrm{Fe}^{3+}$ in these measurements. Therefore the height of the loops does not change with the changing magnetization of the $\mathrm{Er}^{3+}$ ions. There is only a sudden flip of the sign as the magnetization recovers in the opposite direction. Near the SRT the coercive field goes to zero as a result of the decreasing magnetic anisotropy. We find this transition in the temperature interval $86 \mathrm{~K} \lesssim T \lesssim 99 \mathrm{~K}$. These values are very close to those found by measurements of sound velocity ${ }^{26}$ and neutron scattering ${ }^{27}$ in $\mathrm{ErFeO}_{3}$.

\section{SPIN DYNAMICS EXPERIMENT}

To excite and measure the spin dynamics we used a pumpprobe technique. Pulses of $100 \mathrm{fs}$ at a photon energy of $1.55 \mathrm{eV}$ were produced by a Ti:sapphire laser, amplified, and split into a pump and probe part. With a delay line we controlled the delay of the probe with respect to the pump. The pump pulses had an energy of $9 \mu \mathrm{J}$ and were focused to a spot with a full width at half maximum of approximately $120 \mu \mathrm{m}$. They were circularly polarized to create an effective magnetic field in the sample during the pulse by means of the IFE. To avoid light from the pump on the detector, the pump beam deviated slightly from normal incidence on the sample. The probe beam was perpendicular to the sample normal, linearly polarized, about 20 times weaker than the pump, and focused to a spot a bit smaller than that of the pump. The Faraday rotation of the probe pulse polarization stems from the $\mathrm{Fe}^{3+}$ ions only and the rotation is a measure of the component of the $\mathrm{Fe}^{3+}$ magnetization along the direction of the beam. ${ }^{28}$ We aligned the probe polarization along one of the crystal axes of the sample with an accuracy of $\pm 1^{\circ}$. In this way, the effect of linear birefringence on the polarization of light is diminished and the relation between the $\mathrm{Fe}^{3+}$ magnetization and the Faraday rotation is linear. ${ }^{29}$ To ensure that we only measured changes in

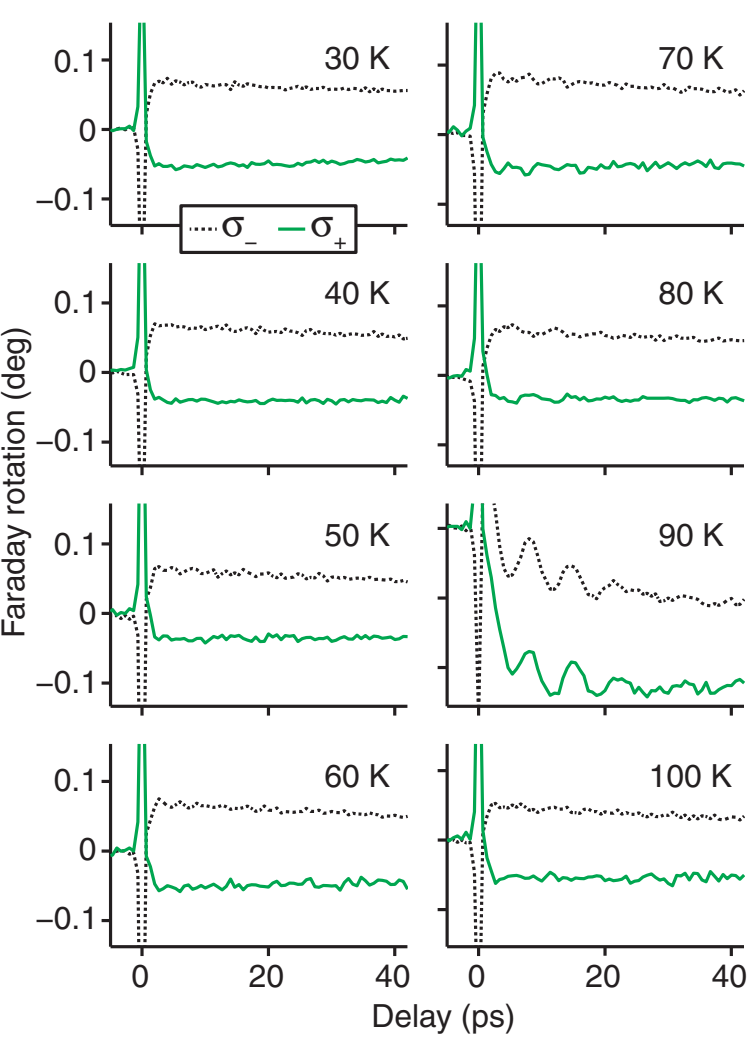

FIG. 3. (Color online) Change of the probe polarization as a function of time delay. The direction of the probe beam is along the $c$ axis of $\mathrm{ErFeO}_{3}$. At $t=0$ the material is excited by a strong laser pulse with circular polarization $\sigma_{-}$(dotted) or $\sigma_{+}$(full). The applied magnetic field during the measurements was $2.3 \mathrm{kG}$ (for field direction see text).

the rotation induced by the pump, we measured the difference between the Faraday rotation with and without the pump.

The temperature of the samples was controlled with a continuous-flow cryostat with a stability of $\pm 0.2 \mathrm{~K}$. With a water cooled electromagnet we could apply fields up to $3 \mathrm{kG}$. The role of the magnetic field in this case was to prepare the sample in a well-defined initial magnetic state for every new measurement at temperatures below and in the SRT region. Therefore, for the $c$ sample, we put the magnetic field along the $a$ axis since this is where the ferromagnetic vector $\mathbf{m}$ points at temperatures below the SRT region [see Fig. 1(b)]. For the $a$ sample we created a small component of the field along the $a$ axis by rotating the magnet by $8^{\circ}$ such that the magnetic field pointed between the $a$ axis and the $b$ axis.

\section{RESULTS AND DISCUSSION}

\section{A. Results}

Figure 3 shows the change of polarization rotation in the probe beam as a function of time delay in the $c$ sample at several temperatures in the range from 30 to $100 \mathrm{~K}$. Measurements for both left- and right-handed circular polarization of the pump pulse and an applied field of $2.3 \mathrm{kG}$ are plotted. We can distinguish three processes, each with their own associated time scale. First of all, we observe a large peak during the overlap of the pump and probe pulses. The sign of the peak 
(a)

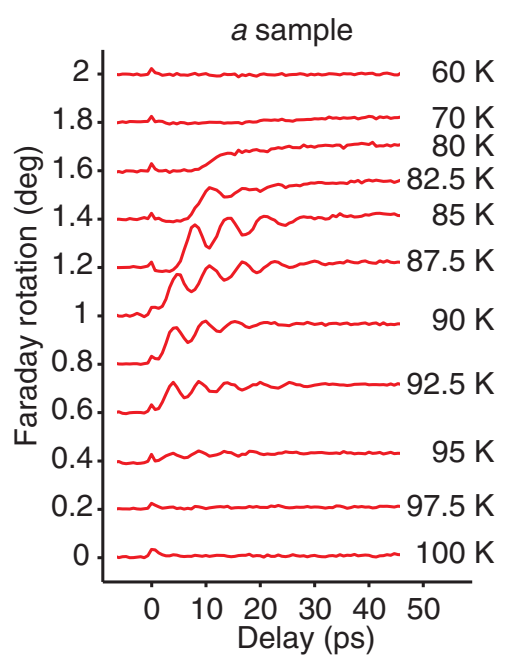

Polarizarion-independent

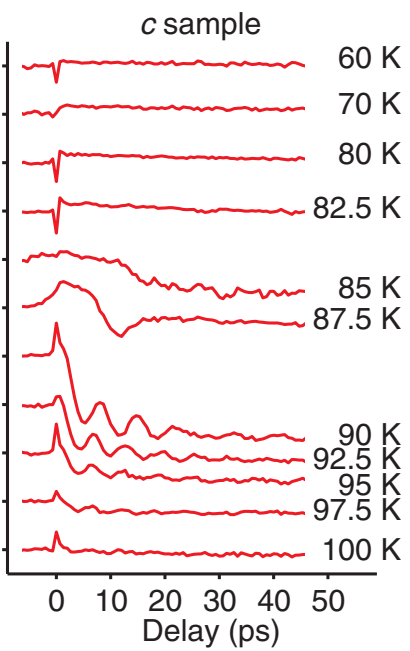

(b) Polarizarion-dependent

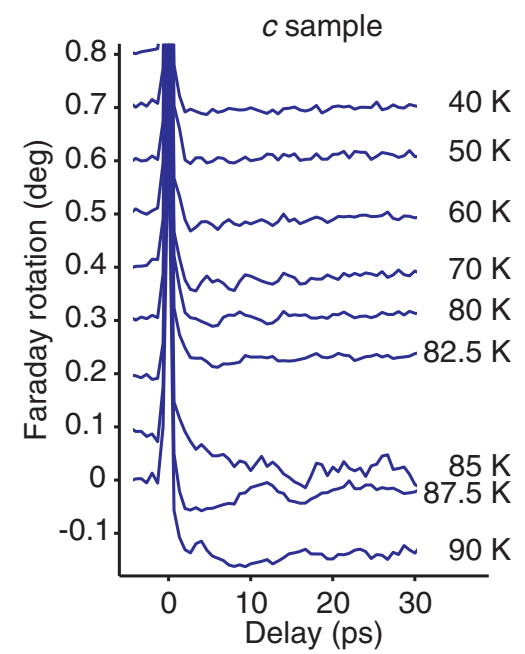

FIG. 4. (Color online) (a) Polarization-independent effect of pump pulse on the Faraday rotation as a function of time delay for temperatures between 60 and $100 \mathrm{~K}$ ( $a$ sample and $c$ sample). These curves are obtained by summation of the curves measured with left- and right-handed circular polarization of the pump. (b) Polarization-independent effect of the pump pulse on the Faraday rotation in the temperature interval 40 to $90 \mathrm{~K}$ ( $c$ sample). Now the curves are the difference between curves measured with left- and right-handed circular polarization. In all graphs curves for different temperatures have been displaced for clarity. The applied magnetic field was $2.3 \mathrm{kG}$.

depends on the helicity of the pump pulse polarization. Second, in the temperature range from 50 to $90 \mathrm{~K}$, after the overlap of the pump and the probe pulses, one can observe oscillations of the magnetooptical signal with a period between 3 and $10 \mathrm{ps}$. Finally, apart from the oscillations, one can distinguish a (gradual) change of the signal toward a new value. The characteristic time ranges from a few to several tens of picoseconds.

The strong peak during the overlap of the pump and probe is a good marker of the zero time delay. It is the result of the population of excited states in the $\mathrm{Fe}^{3+}$ ions and the $\mathrm{Er}^{3+}$ ions. Most of the absorption takes place in the $\mathrm{Fe}^{3+}$ ions and the time scale of the decay of their excited states is much shorter than the pulse. ${ }^{30}$ But also off-resonant pumping of electronic transitions in $\mathrm{Fe}^{3+}$ and $\mathrm{Er}^{3+}$ can change the magnetooptical signal strongly during the overlap of the pump and the probe. ${ }^{31}$ The peak is thus due to a change in the magnetooptical properties of the material rather than a change in the magnetization.

To analyze the behavior of the magnetooptical signal after the action of the pump pulse, we note that the effect of light on the magnetic system can be twofold. First of all, due to the absorption of light and the dissipation of energy in the material, an ultrafast laser excitation leads to an effective generation of phonons and thus serves as an ultrafast heater of the sample. ${ }^{30}$ Near a SRT this temperature increase leads to a change in the direction of the magnetic anisotropy and near a compensation point to a change of the sign of the net magnetization direction. Second, due to the IFE the excitation of a medium with an ultrashort circularly polarized laser pulse can be equivalent to the action of an effective magnetic field pulse. Obviously the latter effect will strongly depend on the polarization of the laser pulse, while laser-induced heating will hardly be influenced by it.
To isolate the polarization-dependent from the polarizationindependent effects we take the difference and the sum, respectively, of the curves measured with opposite circular polarizations of the pump pulse. In Fig. 4(a) the results for the polarization-independent part in the $a$ sample and the $c$ sample are plotted, demonstrating only an effect in or below the SRT. This is consistent with heating as the source of the polarization-independent effect because only near a phase transition heating can effectively bring the spin system out of equilibrium. In the $c$ sample, we also find effects that depend on polarization. These can be seen in the difference curves plotted in Fig. 4(b). The jump of the signal right after the overlap peak is clearly visible. A careful inspection of the curves also reveals some oscillations in the temperature region from 50 to $90 \mathrm{~K}$. From this figure it is not easy to draw conclusions about the source of the polarization-dependent effect. Therefore, we will come back to it after a more detailed analysis. We have not observed any laser-induced spin dynamics below the compensation point. For the $a$ sample the experimental geometry is suitable to observe magnetization reversal upon heating across the compensation point [see Fig. 2(a)]. However, for this the component of the applied magnetic field along the $a$ axis needs to be larger than the coercive field. Clearly, compared to the coercive field of the heated material, this component was too small to switch the magnetization.

\section{B. Laser-induced precession}

The oscillations in the curves shown in Fig. 4 are mainly excited via the heat-driven mechanism. Those excited via the polarization-dependent effect can also be seen, but their amplitude is very small. Both amplitude and frequency appear to be temperature dependent. To analyze these dependencies, 


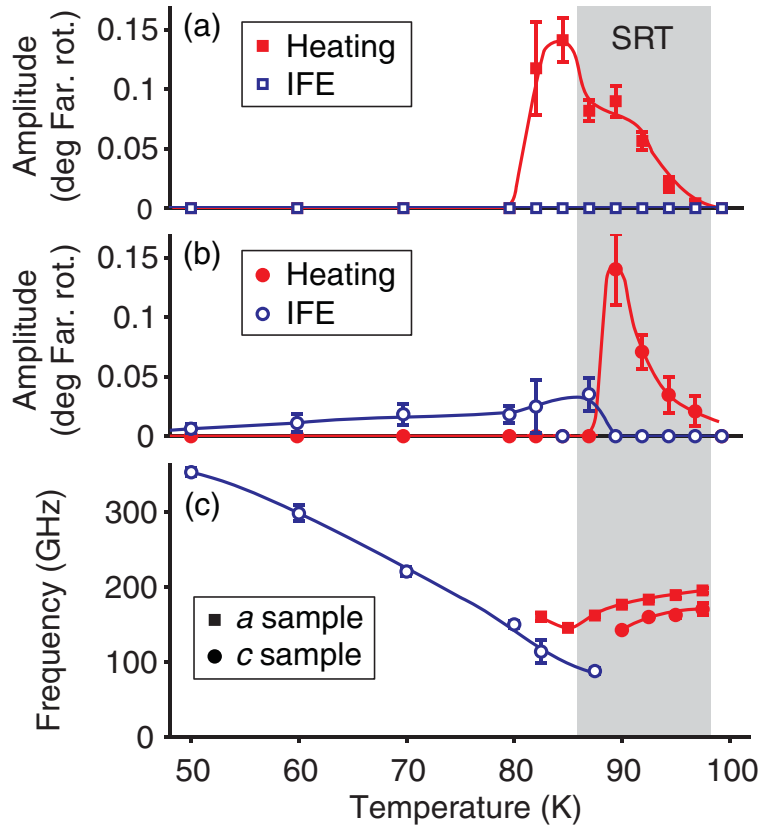

FIG. 5. (Color online) Amplitude of oscillations in the (a) $a$ sample and (b) $c$ sample as a function of temperature. The applied magnetic field was $2.3 \mathrm{kG}$. We distinguish oscillations caused by heating from oscillations caused by the inverse Faraday effect. (c) The frequencies of the oscillations due to heating (filled) and the inverse Faraday effect (open) in the $a$ sample (squares) and the $c$ sample (dots). The error bars are $95 \%$ confidence intervals obtained from the fitting procedure. The lines in these graphs are guides to the eye.

we have fitted the Faraday rotation signals observed at different temperatures using the following function:

$$
\beta(t)=A e^{-t / \gamma_{1}} \cos (2 \pi \nu t-\phi)+B e^{-t / \gamma_{2}}+C .
$$

The first term is the oscillation part and contains the fit parameters $A, v, \phi$, and $\gamma_{1}$. These are, respectively, the amplitude, frequency, phase, and relaxation time of the oscillations. The other two terms approximate the shape of the curve, apart from the oscillations. Fit parameters $B$ and $\gamma_{2}$ are the amplitude and relaxation time of an exponential decay and $C$ is the value at which the signal eventually settles and which is, like the oscillation amplitude, measured in degrees of Faraday rotation.

Temperature dependencies of the amplitude $A$ and the frequency $v$ of the oscillations extracted from the fits are plotted in Fig. 5. The frequencies of all observed oscillations are in very good agreement with the frequency of the quasiferromagnetic resonance (qFMR) mode in $\mathrm{ErFeO}_{3}$ (Ref. 32). This means that the oscillations in Fig. 4(b) are now also identified as an excitation of the spin system. Because they depend on the helicity of the pump polarization, we can conclude that they are excited via the IFE. The temperature dependence of the oscillation amplitudes is consistent with this picture. First of all, the oscillations triggered via heating are excited only if the temperature of the sample is in or below the SRT region. The oscillations excited via the IFE appear also below or in the very beginning of the SRT region. Here the effective magnetic field generated by the circularly polarized light is perpendicular to the ferromagnetic vector and can thus trigger the qFMR mode. Above the SRT the effective magnetic field is parallel to the ferromagnetic vector and thus the qFMR mode can not be excited via the IFE. ${ }^{3,33}$ In principle, in $\mathrm{ErFeO}_{3}$ one can also expect to find a quasi-antiferromagnetic (qAFMR) mode. However, this mode cannot be excited by heating and is therefore of limited interest in the current investigation. If the mode would be somehow excited, it will not be visible in our data because its frequency is in the order of $1 \mathrm{THz}$.

The amplitudes and frequencies in the $c$ sample appear to be shifted a few Kelvin to higher temperatures compared to those in the $a$ sample. This is likely connected with small differences in the mounting of the samples. Note also that the temperature region in which we have been able to excite oscillations by heating is a bit narrower in the $c$ sample. At temperatures just below the reorientation region, these oscillations are only visible in the $a$ sample. In the $c$ sample the curves corresponding to these temperatures have a strange shape [see Fig. 4(a)]. We think this is the result of interplay between the IFE and the applied magnetic field in the determination of the spin reorientation direction. Studies of this effect will be published elsewhere.

In principle, one would expect the frequency of the oscillations to be the frequency of the qFMR mode at the temperature of the heated material. We note that in the $a$ sample, at $82.5 \mathrm{~K}$ and for an applied field of $2.3 \mathrm{kG}$, the spin oscillations excited by heating have a frequency belonging to a temperature lower than that of the SRT (see Fig. 5). If our material would indeed have this temperature, it would be impossible to excite spin oscillations by heating. The same was observed in $\mathrm{TmFeO}_{3}$ (Ref. 8). This can be explained if we take the Gaussian profile of the pump pulse into account. This profile results in a temperature gradient with a maximum temperature in the center of the pump spot. Near the edge of the pump spot, the spins are not brought out of equilibrium by the pump itself, but by their neighbors. Assuming the oscillations in the area are in phase, the resulting qFMR mode frequency will be slightly lowered.

\section{Ultrafast spin reorientation}

Apart from the oscillations, the overall magnetooptical signal eventually settles on a new value. In the case this change is driven by heating, the process can take up to 40 ps to complete. However, in Fig. 4(b), which shows the polarization-dependent effect, this process is completed already during overlap. We first consider the total change, from the pre-overlap value to the end value, which is given by the parameter $C$. In Fig. 6 the temperature dependence of $C$ is plotted for both samples and for applied magnetic fields of 2.3 and $-2.3 \mathrm{kG}$.

In the case the total change is due to heating, the parameter $C$ corresponds to the reorientation amplitude of the spin system. This is supported by the fact that in both the $a$ sample and the $c$ sample the value switches sign if the applied magnetic field is reversed and by the fact that in the $a$ sample the highest value is reached at the lower bound of the SRT. In the $c$ sample the reorientation amplitude shows a kink at $87.5 \mathrm{~K}$. This coincides with the strange shape of the corresponding curves in Fig. 4(a) discussed earlier. 


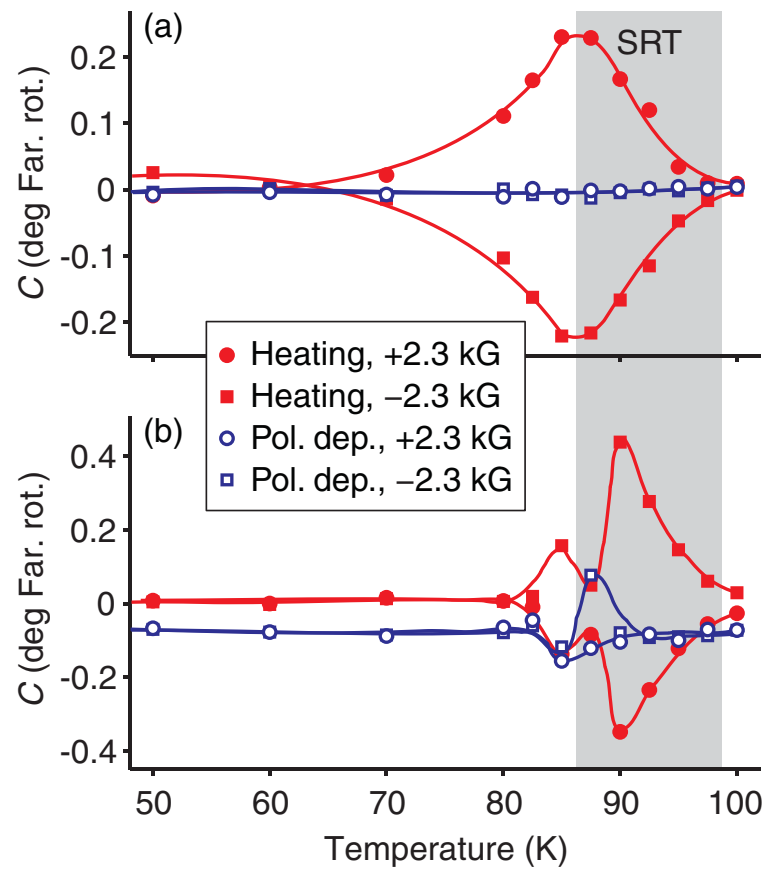

FIG. 6. (Color online) Total change of the Faraday rotation from the pre-overlap value to the value at which the magnetooptical signal settles (parameter $C$ ), as a function of temperature in (a) the $a$ sample and (b) the $c$ sample. Polarization-dependent (open) and the heatinduced (filled) effects of the pump pulse are plotted. The applied magnetic field was $2.3 \mathrm{kG}$ (dots) and $-2.3 \mathrm{kG}$ (squares). Because reversing the applied magnetic field has very little influence on the polarization-dependent effect, these two data sets are almost on top of each other. The lines are guides to the eye.

In the $c$ sample there is also a total change due to the presence of a polarization-dependent effect [see Fig. 6(b)]. The value of $C$, around $0.08^{\circ}$ Faraday rotation, is hardly influenced by temperature or the sign of the applied magnetic field. This is in strong contrast with the temperature dependence of the magnetic structure of $\mathrm{ErFeO}_{3}$, which experiences dramatic changes in the studied temperature range. Therefore, it is natural to suggest that this polarization-dependent effect is not associated with a change in the spin system.

In contrast with the outcome of similar experiments in other RE orthoferrites, the heat-induced spin reorientation in $\mathrm{ErFeO}_{3}$ [see Fig. 4(a)] appears to be significantly slower than a quarter of the period of the antiferromagnetic precession. ${ }^{4,8}$ This is interesting because it implies that in our experiments the reorientation is probably limited by the speed of the change in magnetic anisotropy direction rather than the spin precession period. The magnetic anisotropy in RE orthoferrites depends strongly on the populations of the RE $4 f$ electrons in the different sublevels of the ground multiplet. ${ }^{11,12}$ Considering the slow speed of reorientation in $\mathrm{ErFeO}_{3}$, it is unlikely that a photo-induced anisotropy change (i.e., a direct excitation of these electrons) plays a large role. The repopulation must therefore be driven by thermal excitation of the RE $4 f$ electrons. Because of the large spectral width of the pump pulse compared to the narrow line widths of electronic transitions in $\mathrm{Er}^{3+}$, most of the absorption of the $1.55 \mathrm{eV}$ photons takes place in the $\mathrm{Fe}^{3+}$ ions. To get a reorientation, the energy then needs
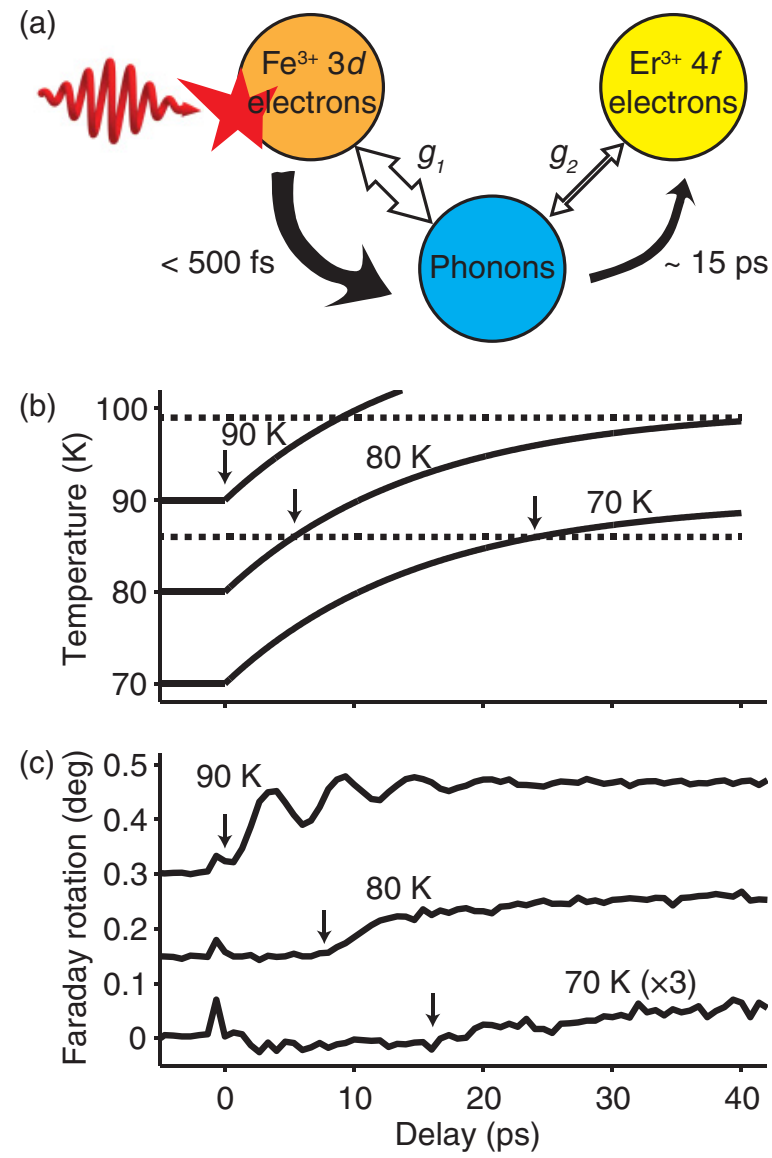

FIG. 7. (Color online) (a) Following the ultrafast laser excitation of $\mathrm{ErFeO}_{3}$, energy flows from the excited $\mathrm{Fe}^{3+} 3 d$ electrons to the $\mathrm{Er}^{3+}$ $4 f$ electrons via the lattice. Due to the strong coupling between the $\mathrm{Fe}^{3+} 3 f$ electrons and the lattice, most of the energy of the excitation will reach the lattice within $500 \mathrm{fs}$. The coupling between the lattice and the $\mathrm{Er}^{3+} 4 f$ electrons is much weaker. Therefore this coupling is the bottleneck in the energy flow. (b) Heating of the $\mathrm{Er}^{3+} 4 f$ electrons due to the coupling to a warmer lattice from three different starting temperatures: 70,80 , and $90 \mathrm{~K}$. At time $t=0$ the temperature of the lattice is instantaneously increased by $20 \mathrm{~K}$. One can see that the reorientation starts at a later point in time (arrows), which depends on the difference between the initial temperature and the temperature at which the spin reorientation transition starts (lower dashed line). (c) Change of the Faraday rotation in the probe pulse as a function of time delay in the $a$ sample for three different temperatures. Note that the start of the reorientation is delayed (arrows) in a similar way as in the model.

to move from the excited $\mathrm{Fe}^{3+} 3 d$ electrons to the $\mathrm{Er}^{3+} 4 f$ electrons [see Fig. 7(a)]. Because the electronic transitions excited in the $\mathrm{Fe}^{3+}$ ions are broad, ${ }^{23}$ thermal equilibrium with the lattice is expected to be reached within a few hundred femtoseconds, like in similar materials. ${ }^{30}$ Therefore, the energy transfer from the lattice to the $\mathrm{Er}^{3+} 4 f$ electrons, via the electron-phonon interaction, appears to be the bottleneck.

This energy transfer from the laser excitation to the $\mathrm{Er}^{3+} 4 f$ electrons via the lattice can be simulated with a simple model in which we make the following assumptions: the heating of the lattice yields a sudden temperature increase at $t=0$, heat conduction through the material does not play a role at this time 

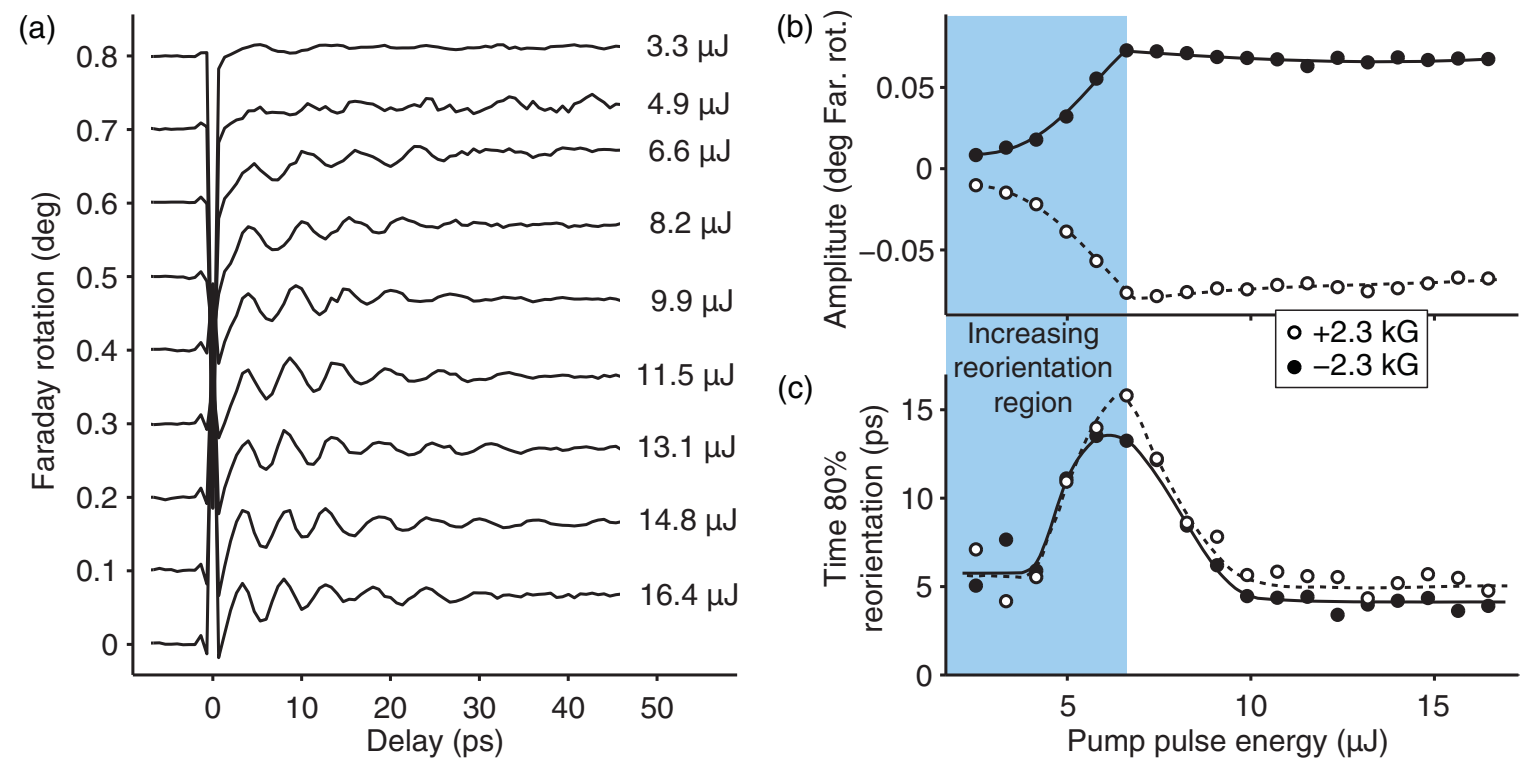

FIG. 8. (Color online) (a) Change of the Faraday rotation in the probe pulse as a function of time delay in the $a$ sample at $90 \mathrm{~K}$ for different pump pulse energies. (b) Reorientation amplitude and (c) reorientation time as a function of pump pulse energy at $90 \mathrm{~K}$ for applied magnetic fields of 2.3 and $-2.3 \mathrm{kG}$. The reorientation time is expressed as the time to reach $80 \%$ of the final reorientation value. The part of the graph where the reorientation amplitude is still increasing, is colored blue. The lines are guides to the eye.

scale, the specific heat capacity of the $\mathrm{Er}^{3+} 4 f$ electrons $C_{e}$ is negligible compared to the lattice specific heat capacity $C_{l}$, and the temperature dependences of $C_{e}$ and the electron-phonon coupling constant $g_{2}$ near the SRT region can be neglected. The process is then governed by the equation:

$$
C_{e} \frac{d T_{e}}{d t}=-g_{2}\left(T_{e}-T_{l}\right),
$$

in which $T_{l}$ and $T_{e}$ are the temperatures of the lattice and the RE $4 f$ electrons, respectively. In total, four parameters are left: the initial temperature, the temperature increase $\Delta T$, and the material properties $g_{2}$ and $C_{e}$.

In Fig. 7(b) the results of the model with an initial heating $\Delta T=20 \mathrm{~K}$ and $\frac{C_{e}}{g_{2}}=15 \mathrm{ps}$ are shown for three different initial temperatures: 70,80 , and $90 \mathrm{~K}$. One can see that, if the initial temperature of the material lies below the SRT, it first needs to warm up to the lower bound of the transition before the reorientation can start. These delays are present both in the simulation and in the measurements [see Figs. 7(b) and 7(c)]. Note that the $y$ axes in both graphs are different. It is not straightforward to accurately determine the temperature increase from the Faraday rotation of the probe. Factors responsible for this are a slightly nonlinear behavior of the spin rotation with temperature, ${ }^{34}$ an increase in the magnetization by $70 \%$ in the interval of the SRT, ${ }^{34}$ the nonlinear relationship between the rotation angle and the component of magnetization that we measure and finally, the inhomogeneous fluence across the sample thickness due to absorption. Nevertheless, the qualitative agreement between the model and the measurements is striking.

The model also predicts that a large temperature difference leads to very rapid heating [see Eq. (2)] and this means that the reorientation speed can be controlled by the laser fluence. Despite the fact that this fluence differs for different places in the sample, it is everywhere proportional to the laser pulse energy. The response of the spin system in the $a$ sample as a function of time delay for different pump pulse energies at $90 \mathrm{~K}$ is plotted in Fig. 8(a). To quantify the results, the reorientation time is defined as the time it takes for the Faraday rotation to reach $80 \%$ of the final reorientation value $C$. The results for the reorientation amplitude and the reorientation time are plotted in Figs. 8(b) and 8(c), respectively. In the region below $6.6 \mu \mathrm{J}$, the reorientation amplitude is still increasing with increasing pump pulse fluence. Because there the reorientation time is not a good indicator to compare the speed, this region is colored blue. For higher pulse energies, we can indeed see a rapid decrease of the reorientation time with increasing pulse fluence. Around $10 \mu \mathrm{J}$ it levels off at a value of $5 \mathrm{ps}$, which is close to a quarter of the antiferromagnetic precession period, as was found previously in $\mathrm{TmFeO}_{3}$ (Ref. 4).

The final two parameters in Eq. (2) are related to the material. The SRT in $\mathrm{TmFeO}_{3}$ is very similar to the one in $\mathrm{ErFeO}_{3}$ and occurs in a similar temperature range. We can also assume that the electron heat capacities in $\mathrm{Er}^{3+}$ and $\mathrm{Tm}^{3+}$ differ only minimally. However, the electron-phonon coupling for $\mathrm{Er}^{3+}$ is significantly weaker than for $\mathrm{Tm}^{3+}$. This is evident from the narrow line widths of the electronic transitions of the $\mathrm{Er}^{3+}$ ions in $\mathrm{ErFeO}_{3}$ (Ref. 24) and is consistent with the variation of the electron-phonon coupling in the trivalent lanthanide ions found in other crystals. ${ }^{15}$ We think this is the most reasonable explanation for the fact that a clear delay between the overlap and the start of reorientation is observed in $\mathrm{ErFeO}_{3}$ (see Fig. 7) and not in $\mathrm{TmFeO}_{3}$ (Refs. 4 and 8).

\section{CONCLUSION}

With 100-fs laser pulses we have excited spin dynamics in $\mathrm{ErFeO}_{3}$. The effective magnetic field during the pulse (IFE) led to excitation of the qFMR mode. We mainly used the laser pulse for rapid heating of $\mathrm{ErFeO}_{3}$ across its compensation point 
and spin reorientation transition. Near the compensation point this did not result in any spin dynamics that we could detect. However, near the SRT it resulted in ultrafast spin reorientation and the excitation of the qFMR mode. The reorientation was slower than previously reported for a similar phase transition in $\mathrm{TmFeO}_{3}$. We can attribute this to the weaker electron-phonon coupling of the $\mathrm{Er}^{3+} 4 f$ electrons with the lattice, which creates a bottleneck in the transfer of the excitation energy to these electrons. By varying the laser pulse fluence, we could control the speed of the reorientation.

\section{ACKNOWLEDGMENTS}

We thank A. F. van Etteger and A. J. Toonen for technical support and J. H. Mentink and A. H. M. Reid for discussions. Samples used in this study were provided by A. M. Balbashov under INTAS Project No. 05-1000008-8112. This work was supported by the Foundation for Fundamental Research on Matter (FOM), The Netherlands Organization for Scientific Research (NWO) and the Russian Foundation for Basic Research (RFBR).
${ }^{1}$ A. Kirilyuk, A. V. Kimel, and T. Rasing, Rev. Mod. Phys. 82, 2731 (2010).

${ }^{2}$ E. Beaurepaire, J.-C. Merle, A. Daunois, and J.-Y. Bigot, Phys. Rev. Lett. 76, 4250 (1996).

${ }^{3}$ A. V. Kimel, A. Kirilyuk, P. A. Usachev, R. V. Pisarev, A. M. Balbashov, and T. Rasing, Nature (London) 435, 655 (2005).

${ }^{4}$ A. V. Kimel, A. Kirilyuk, A. Tsvetkov, R. V. Pisarev, and T. Rasing, Nature (London) 429, 850 (2004).

${ }^{5}$ F. Hansteen, A. V. Kimel, A. Kirilyuk, and T. Rasing, Phys. Rev. Lett. 95, 047402 (2005).

${ }^{6}$ C. D. Stanciu, F. Hansteen, A. V. Kimel, A. Kirilyuk, A. Tsukamoto, A. Itoh, and T. Rasing, Phys. Rev. Lett. 99, 047601 (2007).

${ }^{7}$ K. Vahaplar, A. M. Kalashnikova, A. V. Kimel, D. Hinzke, U. Nowak, R. Chantrell, A. Tsukamoto, A. Itoh, A. Kirilyuk, and T. Rasing, Phys. Rev. Lett. 103, 117201 (2009).

${ }^{8}$ A. V. Kimel, C. D. Stanciu, P. A. Usachev, R. V. Pisarev, V. N. Gridnev, A. Kirilyuk, and T. Rasing, Phys. Rev. B 74, 060403 (2006).

${ }^{9}$ J. P. van der Ziel, P. S. Pershan, and L. D. Malmstrom, Phys. Rev. Lett. 15, 190 (1965).

${ }^{10}$ A. V. Kimel, B. A. Ivanov, R. V. Pisarev, P. A. Usachev, A. Kirilyuk, and T. Rasing, Nat. Phys. 5, 727 (2009).

${ }^{11}$ R. L. White, J. Appl. Phys. 40, 1061 (1969).

${ }^{12}$ A. I. Belyaeva and K. V. Baranova, Izv. Ross. Akad. Nauk, Ser. Fiz. 73, 1117 (2009) [Bull. Russ. Acad. Sci.: Phys. 73, 1056 (2009)].

${ }^{13}$ R. Faulhaber, S. Hüfner, E. Orlich, and H. Schuchert, Z. Phys. 204, 101 (1967).

${ }^{14}$ A. P. Malozemoff, J. Phys. Chem. Solids 32, 1669 (1971).

${ }^{15}$ A. Ellens, H. Andres, M. L. H. ter Heerdt, R. T. Wegh, A. Meijerink, and G. Blasse, Phys. Rev. B 55, 180 (1997).

${ }^{16}$ M. Aeschlimann, A. Vaterlaus, M. Lutz, M. Stampanoni, and F. Meier, J. Appl. Phys. 67, 4438 (1990).

${ }^{17}$ C. D. Stanciu, A. Tsukamoto, A. V. Kimel, F. Hansteen, A. Kirilyuk, A. Itoh, and T. Rasing, Phys. Rev. Lett. 99, 217204 (2007).

${ }^{18}$ D. Treves, J. Appl. Phys. 36, 1033 (1965).
${ }^{19}$ W. J. Tabor, A. W. Anderson, and L. G. V. Uitert, J. Appl. Phys. 41, 3018 (1970).

${ }^{20}$ A. M. Balbashov and S. K. Egorov, J. Cryst. Growth 52, 498 (1981).

${ }^{21}$ K. A. Wickersheim and R. A. Lefever, J. Chem. Phys. 36, 844 (1962).

${ }^{22}$ D. L. Wood, L. M. Holmes, and J. P. Remeika, Phys. Rev. 185, 689 (1969).

${ }^{23}$ P. A. Usachev, R. V. Pisarev, A. M. Balbashov, A. V. Kimel, A. Kirilyuk, and T. Rasing, Fiz. Tverd. Tela 47, 2200 (2005) [Sov. Phys. Solid State 47, 2292 (2005)].

${ }^{24}$ A. Ellens, H. Andres, A. Meijerink, and G. Blasse, Phys. Rev. B 55, 173 (1997).

${ }^{25}$ Landolt-Börnstein New Series Group III, edited by H. P. J. Wijn, (Springer-Verlag, Berlin, 1981), vol. 27f3, pp. 138-142.

${ }^{26}$ Y. B. Bazaliy, L. T. Tsymbal, G. N. Kakazei, A. I. Izotov, and P. E. Wigen, Phys. Rev. B 69, 104429 (2004).

${ }^{27}$ S. M. Shapiro, J. D. Axe, and J. P. Remeika, Phys. Rev. B 10, 2014 (1974).

${ }^{28}$ A. V. Zenkov, B. B. Krichevtsov, A. S. Moskvin, K. M. Mukimov, R. V. Pisarev, and M. M. Ruvinshtěn, Zh. Eksp. Teor. Fiz. 96, 1397 (1989) [Sov. Phys. JETP 69, 792 (1989)].

${ }^{29}$ S. R. Woodford, A. Bringer, and S. Blügel, J. Appl. Phys. 101, 053912 (2007)

${ }^{30}$ A. V. Kimel, R. V. Pisarev, F. Bentivegna, and T. Rasing, Phys. Rev. B 64, 201103 (2001).

${ }^{31}$ J. H. Versluis, A. V. Kimel, V. N. Gridnev, D. R. Yakovlev, G. Karczewski, T. Wojtowicz, J. Kossut, A. Kirilyuk, and T. Rasing, Phys. Rev. B 80, 235326 (2009).

${ }^{32}$ A. Balbashov, G. Kozlov, A. Mukhin, and A. Prokhorov, High frequency processes in magnetic materials (World Scientific, Singapore, 1995), Chap. 2, pp. 56-98.

${ }^{33}$ R. M. White, R. J. Nemanich, and C. Herring, Phys. Rev. B 25, 1822 (1982).

${ }^{34}$ Y. B. Bazaliy, L. T. Tsymbal, G. N. Kakazei, and P. E. Wigen, J. Appl. Phys. 95, 6622 (2004). 